\title{
Research in Gold Mining and Metallurgy
}

\section{NEW TECHNIQUES TO INCREASE EFFICIENCY AND PRODUCTIVITY}

\author{
W. S. Rapson \\ Chamber of Mines Research Organisation, Johannesburg
}

\begin{abstract}
Co-operative research carried out by the Chamber of Mines on behalf of its gold-mining members is mainly directed towards increasing the efficiency of mining and extractive metallurgical operations. Much emphasis is being given to the development of new techniques that might make possible the economic recovery of gold ore from greater depths or from lower grade deposits.
\end{abstract}

The annual world demand for gold for industrial purposes alone is now running at around 1,300 metric tons. Comparison of this figure with the estimated level of the free world production of newly mined gold - some 1,270 tons - underlines the keen interest that users and manufacturers of gold everywhere have in the future supply of the metal.

Over 70 per cent of this newly mined gold is produced by the South African mines as the main product of their working of the reefs of the Witwatersrand series. These reefs, deposited some 2,500 million years ago around the edges of an inland sea, appear as outcrops or sub-outcrops in an arc extending some 250 to 300 miles from Evander in the Eastern Transvaal to Welkom in the Orange Free State.

Mining operations, which began in the vicinity of Johannesburg in 1886, have been expanded steadily as extensions of the reefs have been identified, and there are now forty mines operating on this "gold crescent", The reefs characteristically dip to the south from the outcrop and as mining proceeds must be pursued to progressively greater depths. The stage has now been reached when many of these mines are operating at depths in excess of one mile or 1,500 metres, with some operations going on at depths of the order of two miles or 3,000 metres. Nowhere else in the world is mining being extended to such depths on such a scale, so that advances made elsewhere in the science and technology of mining do not always provide solutions to the most critical problems that have to be faced by South African mining engineers.

It thus became obvious about ten years ago that the South African mining industry would have substantially to increase its own research and development effort in the fields of mining science and technology, and accordingly, in 1964, the Chamber of Mines Research Organisation came into being to initiate and direct the necessary large-scale research programme.

At the present time expenditure by the Research Organisation for the gold mining industry is in excess of R2.2 million (say $f_{1} 1 \cdot 1$ million) per annum, these costs being distributed among the gold mining company members of the Chamber of Mines of South Africa. The main research effort is directed towards the development of new knowledge and techniques to make possible the safe and economic recovery of ore from substantially greater depths, or from lower grade deposits. If successful, this work will contribute greatly to a fuller exploitation of the gold-bearing reefs.

Considerable attention is concurrently being given to the improvement of the efficiency of current mining methods and gold recovery processes.

\section{Strata Control}

A major programme of research in the field of rock mechanics has yielded important advances in strata control in mines. The frequency and severity of "rockbursts", due essentially to uncontrolled and sudden failure of highly stressed rock surrounding deep excavations, can now be minimised by planning mines in such a way that peak rock stresses are avoided, and the energy generated by overburdened subsidence into mining excavations is more uniformly and less violently released. Analogue and digital computer programs for planning mines in accordance with sound rock mechanics principles have been developed and are commonly in use on the gold mines.

In addition, the collapse of "hanging-wall" fractured rock into mine excavations close to working faces, where timber support cannot readily be installed, can now be controlled by recently developed 
The surface installations of one of the newer gold mines on the Witwatersrand, Western Deep Levels. Operations began here in 1962, and at the present time a third shaft is being sunk to open up the mining areas at depths hetween 3,500 and 4,000 metres. This will make Western Deep Levels the deepest mine in the world, but the inereasing depths involve great technical problexas in mining methods, in the safety of workers, and in ventilation and cooling

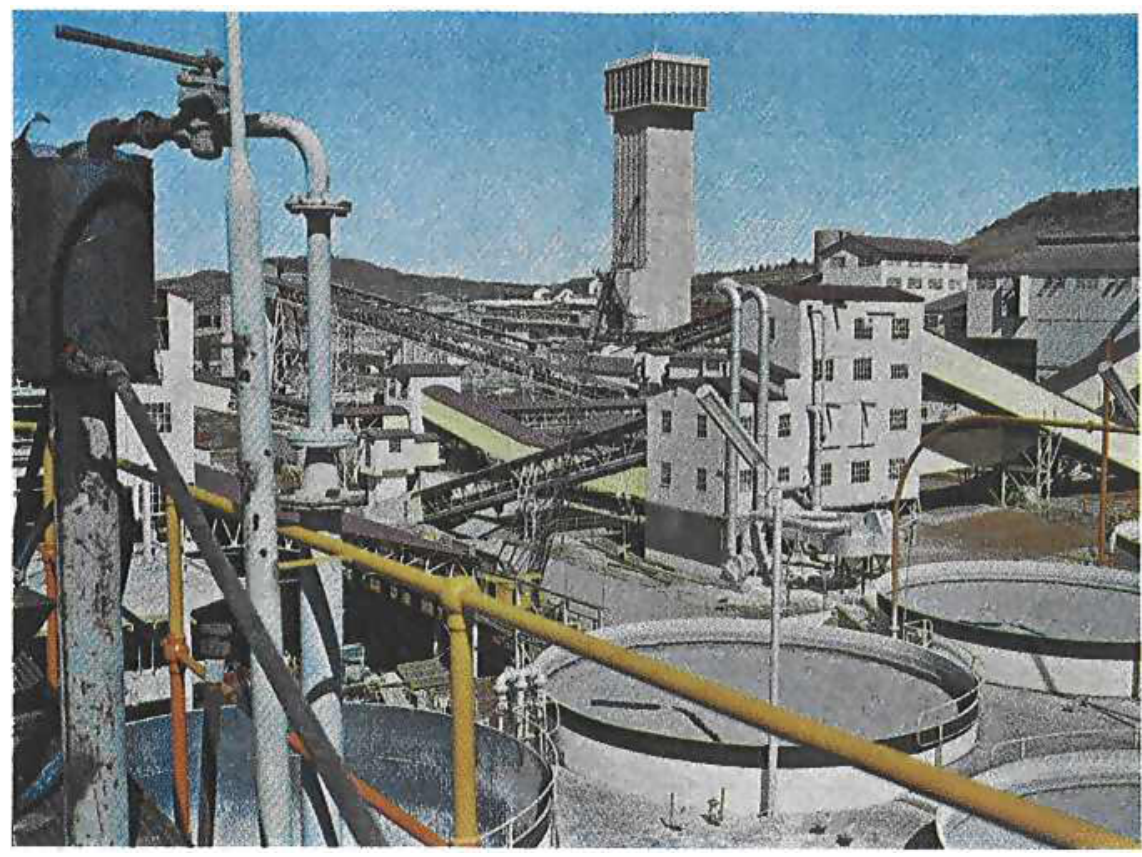

rapid-yielding hydraulic steel props. This development makes possible a significant improvement in productivity, and in particular, provides a major improvement in mining safety.

\section{Selective and Continuous Mining by Rock-cutting}

In a large proportion of the reefs of the Witwatersrand series virtually all the gold is concentrated in a thickness of less than twenty-five centimetres and often in as little as five centimetres. The established method of drilling and blasting results in the removal, not only of the reef, but also of the barren rock that surrounds it to a thickness of about a metre. The reef is thus considerably diluted with barren rock which is hoisted to the surface and milled together with the gold-bearing reef. In addition, the necessity of evacuating mine workings before each blast - and for some time afterwards to allow fume and dust to clear - constrains current mining to a cyclic procedure, with all the limitations on productivity that such procedures are known to impose on any process.

Since a system of continuous extraction of the gold reef without dilution by waste rock would clearly result in a marked increase in the efficiency of underground operations, it is towards this goal that a great deal of effort is being directed.

The development of new techniques for selectively excavating the reefs from the very hard and abrasive quartzites in which they occur is being pursued in several directions. The method offering the best prospects of success in the near future involves cutting a groove in the rock above and below the reef

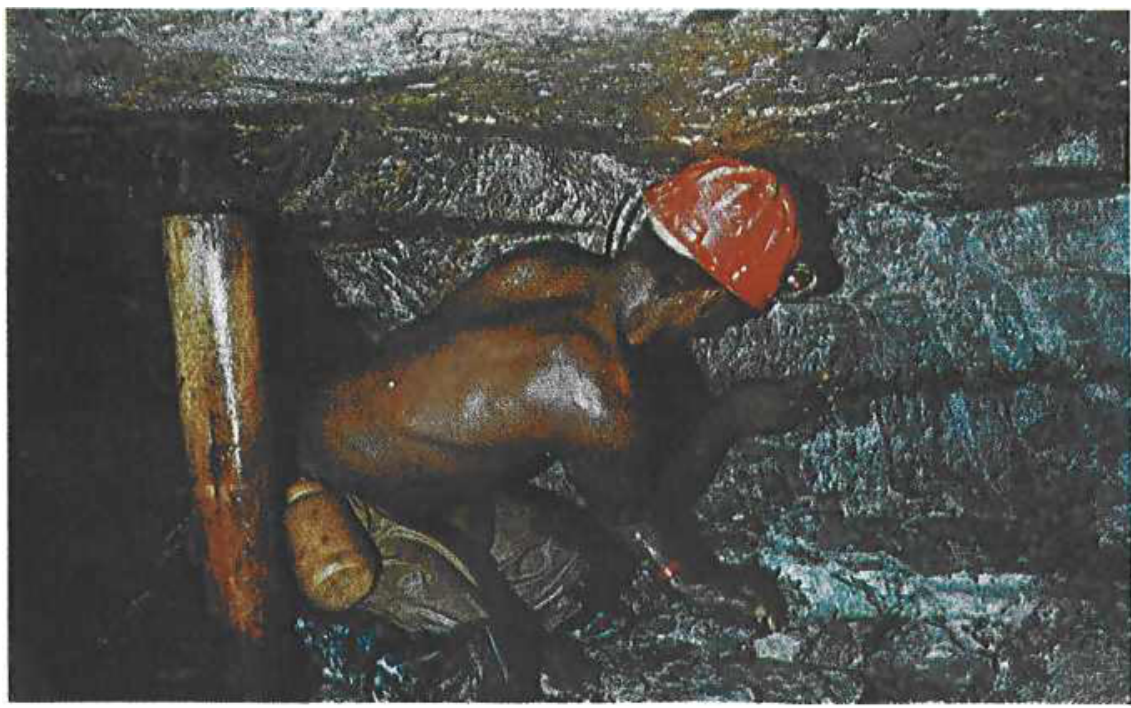

In the Carbon Leader reef virtually all the gold is concentrated in a thickness of the few centimetres shown between this miner's two fingers, yet an average thickness of about one metre has to be blasted out and brought to surface. Two-thirds of the South African gold now produced comes from reefs of this type and research is therefore being directed towards methods for the selective and continuous extraction of a much smaller thickness containing the reef 


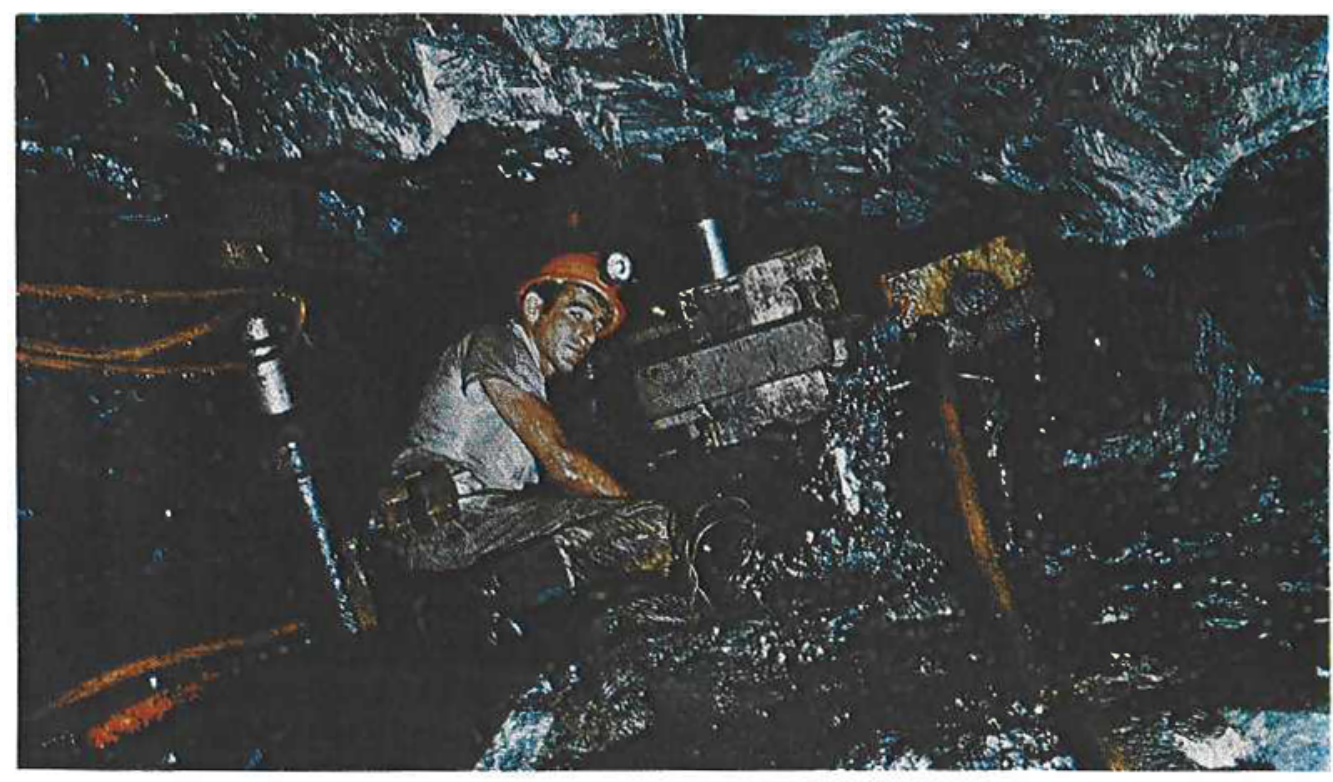

To reduce the thickness of rock to be mined and treated, and to supersede the present technique of blasting, the method of rock-cutting that offers the best prospects of success in the near future involves cutting a groove above and below the reef with a specially-hard-tipped drag bit mounted on a relatively lightweight machine. This experimental rock-cutting machine is removing the narrow gold-bearing portion from the rock face on the right. As the larren roek ahove and helow the reef is packed behind it on the left, the miners, in effect, go forward in a moving tunnel. Support problems are reduced, and mining is made more feasible at depths at which the greater widths of open excavation which result from the use of explosives cause severe problems

with a carbide drag bit. Using machines equipped with such bits, the face can be advanced continuously without the use of explosives, while the amount of rock to be removed to the surface is reduced to onehalf or less. This technique also reduces the problems of support and ventilation, since these are a function of the width of rock removed, and shows every indication of making possible mining at depths which could not be considered with explosive rock-breaking methods.
It has also been found that the rock at the base of the cut can be weakened ahead of the cutting tool by thermal stresses generated by a relatively low power flame or other high temperature source. This modification promises to make the cutting of the hardest rocks much easier.

In addition to rock-cutting machines two other rock-breaking devices are being developed. One is a swing-hammer machine, comprising a power-driven rotor supporting a number of heavy, free swinging,

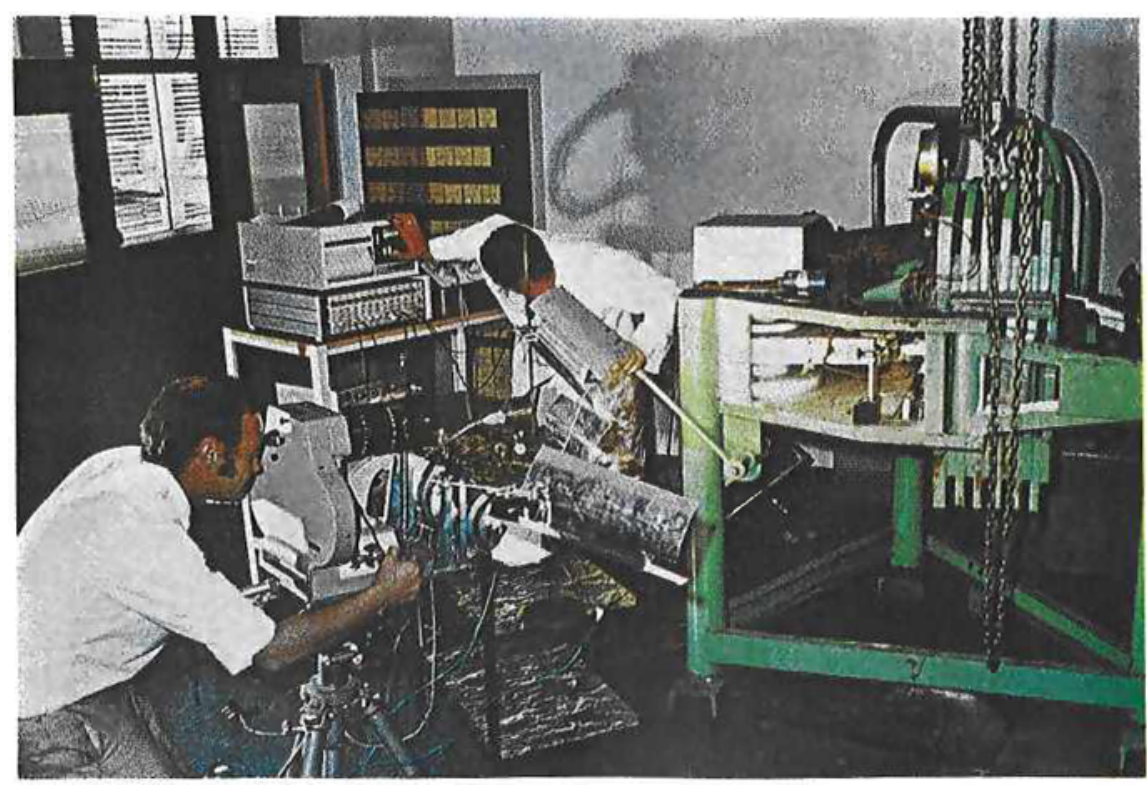

Experimental work on the new method of drag-bit rockcutting, using cubical rock specimens of $30 \mathrm{~cm}$ side, includes the measurement of tangential cutting forces, normal penetrating forces and bit acceleration and their recording on a high frequency oscillograph over the full length of the $30 \mathrm{~cm}$ cut. This work provides data essential for the optimum design of bits and machines 
The simulation of mining activities on digital computers and by analogues now makes it possible to examine practically every operation over a wide range of controlled conditions at great speed and relatively low cost. Here engineers are using an electrical analogue to decide on the best sequence of mining a large block of gold-bearing ore about 300 metres wide by 1,000 metres long in one of the deeper mines. The pins in the board represent unmined areas. For each mining sequence the analogue recorls the rock stresses and displacements, and the energy release rates that are involved

tungsten carbide tipped hammers centrifugally driven to strike and chip pieces off the rock. The other known as the shockwave rockbreaker, utilises the high pressures generated by detonation of mixtures of combustible gases with air. By varying the amounts of fuel and air loaded into a re-usable cylinder the amount of energy produced can be adjusted to suit particular rock-breaking needs.

\section{Simulation by Digital Computer}

Developments such as rock-cutting provide the tools to make mining more productive; the next important problem is that of deciding how best to use them. It is not practicable to acquire all the necessary experience underground, but fortunately the simulation of virtually every mining activity has become feasible with the advent of large digital computers. At great speed and relatively small cost, practically any mining activity can now be examined by computer simulation over a wide range of controlled conditions. In this way engineers can gain experience of new situations very quickly and easily, and can arrive at practical solutions to the design of mines and the planning of operations while new ideas can be evaluated with equal facility.

Programming for the simulation of stoping activity was begun in 1969, using practical data on the direction of unit operations detailed to the extent that the time required for the drilling of each hole and for every pass of a scraper is accounted for on the computer program. The simulation of underground and shaft transport systems is presently being brought into operation. These techniques are now being made available to gold mines and promise to facilitate greatly the introduction of new mining techniques and systems.

\section{Environmental and Human Studies}

The need for thermal control in deep-level mines, where rock temperatures are high, contributes significantly to mine operating costs in the provision of cool ventilating air and, ultimately, underground refrigeration. Computer programs covering the optimum distribution of ventilating air and the use of refrigeration in current workings, as well as in

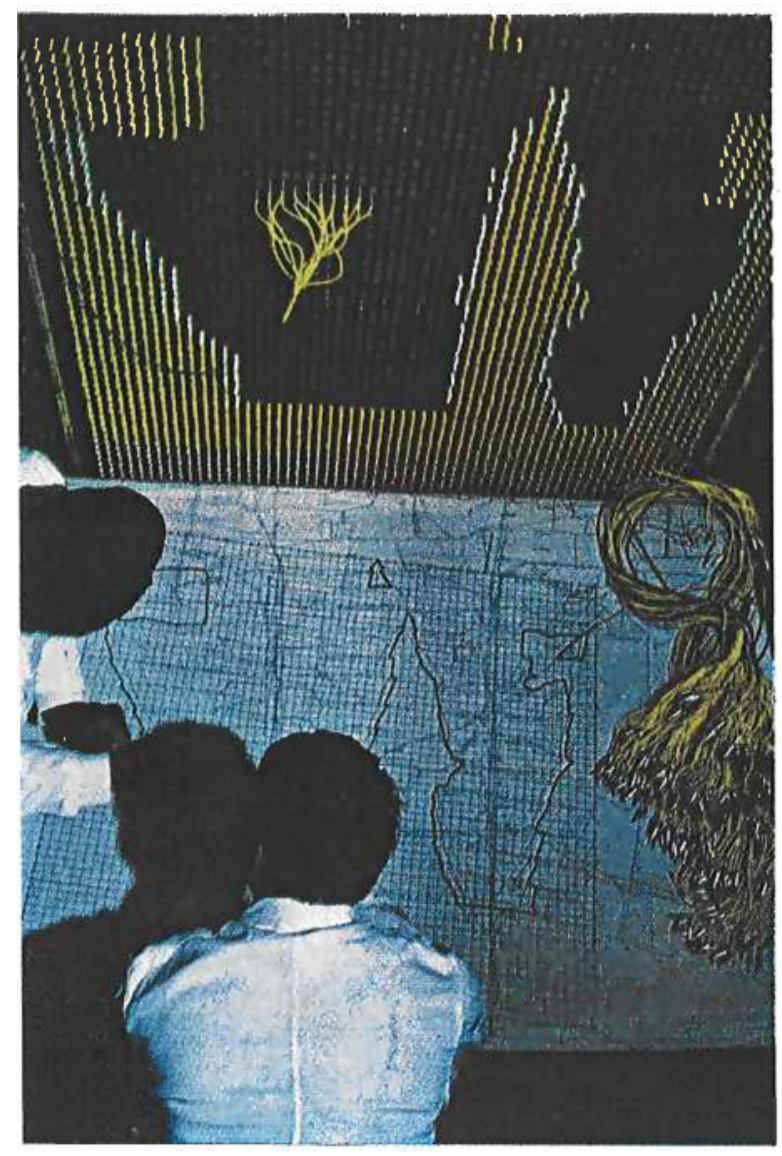

predicting requirements in planned workings, have been developed by the laboratories, and are coming into regular use in mine ventilation departments.

In addition to engineering developments to improve heat stress conditions in mines, the Research Organisation continues to pursue studies of the physiological responses of men required to work in conditions of heat stress. Based on this work, acclimatisation procedures have also been successfully introduced which enable some 150,000 men to work in the hot humid conditions at ultra-deep levels with a minimum of heat strain. As mines go still deeper, acclimatisation and ventilation practices will have to be supplemented in many areas by microclimate cooling, and pilot tests on the effects of wearing pre-frozen jackets, with sealed sachets of water built into them, have been most promising.

\section{Extractive Metallurgy}

Another aim of current research is to integrate, where possible, metallurgical practice more closely with underground operations in order to improve the efficiency of the total activity. In this context the development of a high-capacity centrifugal mill could be of importance. In the centrifugal mill the grinding forces are increased in proportion to the centrifugal force field, so that the physical size of 


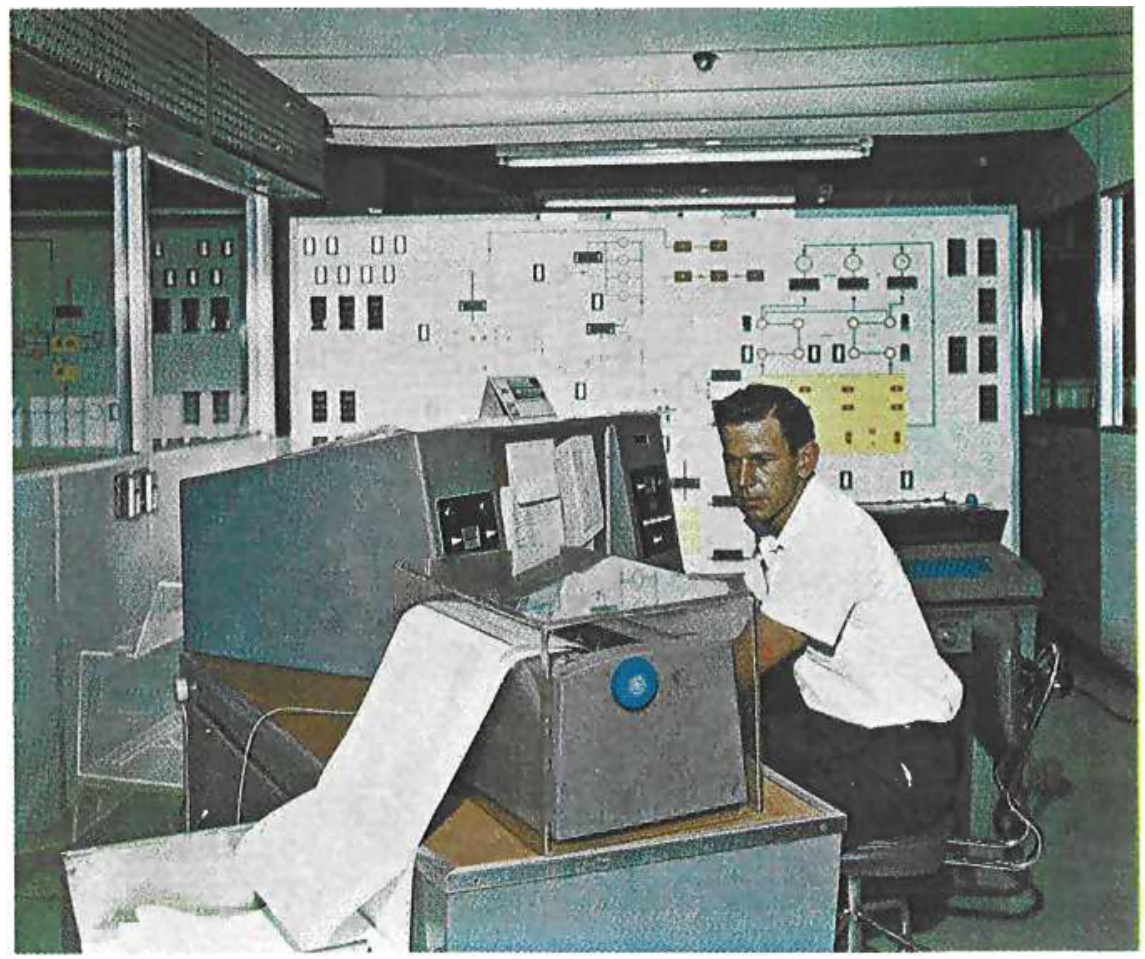

Computer control has been successfully developed on the reduction plant at the Kloot gold mine. This plant mills and treats some 2 million metric tons of gold ore a year, and is entirely automated, the computer program governing every operation including the sequential startup and shut down of motors, conveyors and pumps. Inside the control centre information on the performance of hundreds of individual items of plant is recorded

the mill is reduced for a given duty. A prototype mill has already shown the way to the design of a full scale continuous machine capable of handling several tens of tons of material per hour.

In the old-established cyanide process for dissolution of gold, passivation effects can rapidly reduce the initially high rate of dissolution, and no possibility of overcoming this effect has so far been found. However, in acidic systems no such passivation occurs so that in acid leaches of typical milled ores in which thiourea is used as a complexant, residue values equivalent to those obtained in cyanide solution in 48 hours can be obtained in as short a time as four hours. Methods for prevention of the loss of thiourea by oxidation are being studied with a view to possible pilot scale studies.

At the same time new methods of recovery of gold and silver values from cyanide leach solutions, based upon solvent extraction and ion exchange are being explored. In this connection a countercurrent cyclone/resin-in-pulp system, which could do away with the need for filtration, is of special interest.

\section{Industrial Uses of Gold}

The limiting of the official monetary market for gold and the simultaneous development of an unofficial market has given producers a much increased interest in the industrial use of gold.

Since about 80 per cent of the gold used industrially is absorbed in jewellery manufacture, a major effort is now being devoted to promotion of gold jewellery through International Gold Corporation Limited, established for the purpose.

Development of the use of gold for more truly industrial purposes is unlikely to be affected by promotional techniques, however, and a different approach has been adopted. On the one hand, through the Gold Bulletin an attempt is being made to keep organisations and persons with an existing or potential scientific or industrial interest in gold informed of the many exciting developments in the use of this metal. On the other, support is being given for a limited programme of research in relation to existing or new uses of gold.

Gold producers in South Africa have, therefore, now joined the ranks of the producers of other metals such as nickel, tin and copper who reinforce their marketing activities by technical information services and by co-operative research and development.

In addition to the carrying out of research in its own laboratories the Chamber of Mines contracts out selected aspects of its research to such bodies as the Council for Scientific and Industrial Research and to appropriate departments of universities. It also calls upon its individual members and the mining groups for assistance and collaboration. The Research Organisation has now been in existence for eight years, and this account of its achievements and expectations will, it is hoped, interest industrial scientists in many disciplines, particularly in so far as ensuring the adequate supply of gold for the advanced technologies of the present and future is concerned. 